\title{
Über das Orceïn
}

von

Prof. Karl Zulkowski und Assistenten Karl Peters.

Aus dem chemisch-technologischen Laboratorium der k. k. deutschen technischen Hochschule in Prag.

(Vorgelegt in der Sitzung am 6. Juni 1890.)

Die ältere Färbekunst bediente sich mehrerer, aus verschiedenen Flechten dargestellter Farbmaterialien, deren werthrollster Bestandtheil ein rother Farbstoff, das Orceïn ist.

Schon zu Beginn des 14. Jahrhunderts sind in Europa orceïnhaltige Farbmaterialien erzeugt und angewendet worden, und allem Anscheine nach war diese Kunst schon früher im Oriente bekannt. Trotz dieser frühen und häufigen Anwendung: und den bedentenden Fortschritten, welche die Chemie, - gerade auf dem Gebiete der Farbstoffe - gemacht hat, ist über die Natur des Orceïns sehr wenig bekannt und seine in den Druckschriften angegebene Molecularformel muss jeder Chemiker als unrichtig* bezeichnen.

Robiquet war der erste, welcher aus den Färbeflechten den farbstoffbildenden Bestandtheil, das Orcin abuscheiden verstand, und welcher den daraus erhaltenen Farbstoff mit dem Namen Orceïn bezeichnete. Aus den Analysen von Dumas und Kane berechnete Gerhardt seine Formel und stellte folgende Bildungsgleichung auf:

$$
\mathrm{C}_{7} \mathrm{H}_{8} \mathrm{O}_{2}+\mathrm{NH}_{3}+3 \mathrm{O}=\mathrm{C}_{7} \mathrm{H}_{7} \mathrm{NO}_{3}+2 \mathrm{H}_{2} \mathrm{O} \text {. }
$$

Die Herstellung der drei hauptsächlichsten orceïnhaltigen Farbmaterialien d. s. Orseille, Cadbear, Persio, geschieht in der Hauptsache derart, dass man die Färbeflechten als Ganzes, oder einen mit Kalk oder Ammoniak erhaltenen Auszug der Einwirkung von Ammoniak und Luft aussetzt. Nach längerer Zeit erhält man ein violett anssehendes Farbmaterial, welches je nach 
Umständen als Teig, Flüssigkeit oder in trocknem Zustande in Handel gesetzt wird.

Diese Präparate müssen auf Grund der von uns erlangten Erfahrungen, vorzugsweise violette und blane Ammonverbindungen mehrerer Farbstoffe enthalten, welche in freiem $\mathrm{Zu}$ stande amarantroth, beziehungsweise zwiebelroth und orangegelb erscheinen, daher werden die mit solchen alkalischen Farbmaterialien erbaltenen Färbungen durch Säuren mehr oder weniger verändert.

In Frankreich wurde im Jahre 1857 ein säureechter Flechtenfarbstoff erzengt, welcher seitdem unter dem Namen "französischer Purpur" im Handel erschien. Zu diesem Zwecke wurden die Flechten kalt mit Ammoniak ausgezogen und der Auszug mit einer Säure versetzt, um die Flechtensäuren niederzuschlagen. Diese wurden wieder in Ammoniak gelöst und die Flüssigkeit, sobald sie eine tiefkirschrothe Farbe angenommen hatte, in flachen Gefässen bei 60 bis $70^{\circ} \mathrm{C}$. concentrirt. Aus dieser violett gewordenen Flüssigkeit liess sich der Farbstoff durch Salzsäure herausfällen, der sodann gesammelt, gewaschen und getrocknet wurde. Diesem Purpur soll ein "blassrother" Farbstoff beigemengt sein, den man dadurch entfernte, dass man die ammoniakalische Flüssigkeit mit Chlorcalcium oder Alaun versetzte und den reinen Farbstoff als Kalk- oder Thonerdelack abschied. Diese Lacke mussten selbstverständlich bei ihrem Gebrauche mit einer Säure behandelt werden, um den Farbstoff in Freiheit zu setzen.

Schützenberger ist es gelungen, aus dem Kalklack, durch Behandlung mit Oxalsäure, durch Ausziehen mit siedendem Alkohol und Verdunsten dieser Lösung den rothen Farbstoff krystallisirt zu erhalten.

Ob der französische Purpur Orceïn oder ein anderer verwandter Farbstoff sei, davon lässt die Literatur nichts erkennen; man war unseres Erachtens eher geneigt, das erstere anzunehmen.

Im Jahre 1874 hat Li ebermann ${ }^{1}$ in Gemeinschaft mit H. Troschke die Entstehung des Orceïs studirt und es schien den Genannten nicht unmöglich zu sein, dass die vereinte

1 Berl. Berichte 1874. Seite 247 und 1099. 
Wirkung von Ammoniak und Luft sich gemäss der Gleichung :

$$
\mathrm{NH}_{3}+3 \mathrm{O}=\mathrm{H}_{2} \mathrm{O}+-\mathrm{NO}_{2} \mathrm{H}
$$

durch die der salpetrigen Säure ersetzen liesse.

Die in dieser Richtung von Liebermann ${ }^{1}$ und von Weselsky, ${ }^{2}$ ferner in jünster Zeit von Nietzki und Maeckler ${ }^{3}$ unternommenen Versuche haben gezeigt, dass hiebei ein ganz anderer Farbstoff gebildet wird.

Weselsky's Farbstoff ist das jetzt so benannte Orcirufin, welchem die Formel $\mathrm{C}_{14} \mathrm{H}_{14} \mathrm{NO}_{3}$ zukommt und Liebermann's Farbstoff ist im Wesentlichen nichts Anderes, als unreines Orcirufin. Diese Substanz zeigt in alkalischer Lösung eine prachtvolle Fluorescenz, welche den Orseillefarbstoffen mangelt.

Liebermann fand sodann, dass bei der Einwirkung von Ammoniak und Luft auf Orein nicht ein, sondern zwei Farbstoffe gebildet werden, welchen die. Zusammensetzung $\mathrm{C}_{14} \mathrm{H}_{13} \mathrm{NO}_{4}$ und $\mathrm{C}_{14} \mathrm{H}_{12} \mathrm{~N}_{2} \mathrm{O}_{3}$ zukommt. Der erstere Farbstoff wïrde sich nach der Gleichung:

$$
2 \mathrm{C}_{7} \mathrm{H}_{8} \mathrm{O}_{2}+\mathrm{NH}_{3}+3 \mathrm{O}=\mathrm{C}_{14} \mathrm{H}_{13} \mathrm{NO}_{4}+3 \mathrm{H}_{2} \mathrm{O}
$$

bilden, der zweite durch weitere Einwirkung des Ammoniaks und der Luft auf den ersteren entstehen, wie folgende Gleichung: zeigt :

$$
\mathrm{C}_{14} \mathrm{H}_{13} \mathrm{NO}_{4}+\mathrm{NH}_{3}+\mathrm{O}=\mathrm{C}_{14} \mathrm{H}_{12} \mathrm{~N}_{2} \mathrm{O}_{3}+2 \mathrm{H}_{2} \mathrm{O} \text {. }
$$

Den letzteren sollte man daher reichlicher bei sehr lang davernder Einwirkung des Ammoniaks und der Luft erbalten. Beide Farbstoffe sind angeblich lebhaft kantharidenglänzende a m or phe Substanzen und in ihrem Äussern nicht unterscheidbar. Die prachtvoll purpurnen alkalischen Lösungen waren für die erste Substanz röther, für die zweite blaner. Der zweite Farbstoff war in Alkohol, vorzüglich in ammoniakalischem Wasser schwerer löslich als der erste.

Liebermann gelang es nicht, auf Grund der letzteren Eigenschaft eine Trennung beider Farbstoffe durchzuführen, auch andere Methoden führten nicht zum Ziele; es sind demnach die

1 Berl. Berichte 1874. S. 439 .

2 Berl. Berichte 1875, S. 1649.

3 Berl. Berichte 1890, S. 720. 
analysirten Präparate nicht genügend rein gewesen und die oben angeführten Formeln nur als die wabrscheinlichsten anzusehen. ${ }^{1}$

Die Liebermann'schen Arbeiten haben bis zur jüngsten Zeit keine Ergänzung erfahren und es blieb die Bildnng der Orseillefarbstoffe nach wie vor in Dunkel gehüllt.

Der erstgenannte Verfasser vorliegender Abhandlung hat vor mehreren Jahren das Wasserstoffhyperoxyd versuchsweise angewendet, um in seinen Vorlesungen die Bildung des Orceïns zu zeigen. Dieser Versuch glückte, denn schon in ganz kurzer Zeit entstand dieser Farbstoff aus den angewendeten Lösungen in solcher Menge, dass die Flüssigkeit schliesslich einen körnigkrystallinischen Brei darstellte. Auf Grund dieses Ergebnisses wurde dieser Versuch in grösserem Massstabe und in verschiedenartiger Weise wiederholt und hiedureh einstweilen die Überzeugung gewonnen, dass dieser Process kein glatter sei. Die in der Literatur verzeichnete Eigenschaft, wornach das Orceïn ein amorpher Körper sei; war für das Studium dieses Gegenstandes nicht sehr ermuthigend und der Grund, warum dasselbe erst nach längerer Zeit und unter günstigeren Arbeitsverhältnissen aufgenommen wurde.

$\mathrm{Zu}$ dieser Arbeit wurde reines, künstlich erzengtes Orcin verwendet, welches von H. Trommsdorff in Erfurt bezogen wurde.

Es wurden zuerst Versuche unternommen, in welcher Weise Ammoniak und Luft auf das Orcin einwirken und jene haben unzweideutig das überraschende Ergebniss geliefert, dass hiebei drei Farbstoffe gebildet werden; nämlich:

a) Ein rother Farbstoff in grösster Menge, welcher das vielumstrittene Orcein ist, und welches keinen amorphen, sondern einen krystallinischen Körper darstellt; ferner als Nebenproducte:

b) ein gelber, ebenfalls krystallinischer Farbstoff, und

c) ein amorpher lakmusartiger Farbstoff.

Der Umstand, dass die ersten zwei Farbstoffe krystallinisch sind, hat die Untersuchung dieses Processes sehr gefördert und

1 Berl. Berichte 1875. 1649. 
es ist schwer begreiflich, wie es möglich war, die ausgesprochen krystallinische Beschaffenheit des Orceïns zu übersehen.

Ferner wurden Versuche unternommen die Farbstoffbildung durch Anwendung von Wasserstoffhyperoxyd zu beschleunigen. Diese haben wieder ergeben, dass der Process in der That nur so viele Stunden beansprucht, als man im ersten Falle Tage nöthig hat, und dass derselbe genau dieselben Farbstoffe liefert.

Endlich wurde festgestellt, dass man unter gleichen Bedingungen aus Resorcin keinen brauchbaren orceïnartigen Farbstoff erhalten könne, wohl aber wenn man dasselbe mit Orcin combinirt.

Die zwei krystallinischen Farbstoffe wurden rein dargestellt und deren Molecularformel festgestellt, der dritte Farbstoff aber, liess sich bis jetzt noch nicht rein erhalten, daher seine Zusammensetzung einstweilen fraglich bleibt.

Die Art wie die Versuche durchgeführt, welche Wahrnehmungen hiebei gemacht wurden und zu welchen Ergebnissen dieselben geführt haben, soll im nachfolgenden Abschnitte besprochen werden.

\section{Über die Einwirkung von Ammoniak und Luft auf Orcin.}

Es wurden in grösserem Massstabe zwei Versuche unternommen; bei dem ersteren nahm man

$50 \mathrm{~g}$ krystallisirtes Orcin in etwa $200 \mathrm{~cm}^{3}$ Wasser, und $200 \mathrm{~cm}^{3}$ gewöhnlichem Salmiakgeiste gelöst.

Diese Flìssigkeit kam in einen g'eräumigen Kolben, welcher nur mit einem Becherglase bedeckt wurde. Dieseibe nahm sehr bald eine rothbraune Farbe an und wurde in einigen Tagen intensiv violett, wobei sich auch ein körniger Niederschlag in geringerer Menge bemerkbar machte. Durch Neigen des Kolbens liess sich deutlich eine dünne, braungelbe Schichte bemerken, welche an der Glaswand anhaftete. Die Menge des Niederschlages nalim beim weiteren Stehen sichtlich zu und nach etwa zwei Monaten war das Ganze ein dicklicher Brei.

Der Salmiakgeist hat bei diesem Processe einen doppelten Zweck zu erfüllen: nämlich den Stickstoff zu liefern und das Ganze in Lösung zu erbalten. Man sieht, dass der letztere Zweck 
nur unvollkommen erreicht wird, wenn man sich auf die oben angegebene Mengre von $200 \mathrm{~cm}^{3}$ beschränkt.

Bei dem zweiten Versuche wurden

$50 \mathrm{~g}$ krystallisirtes Orcin in

$500 \mathrm{~cm}^{3}$ Wasser gelöst,

$150 \mathrm{~cm}^{3}$ Salmiakgeist und

$20 \mathrm{~g}$ Ätzkali zugesetzt.

Die Menge des Salmiakgeistes wurde verringert, dagegen etwas Ätzkali zugefügt um die gebildeten Stoffe in Lösung zu erhalten und hiedurch eine bessere Oxydation zu ermöglichen.

Es musste nämlich die Möglichkeit ins Auge gefasst werden, dass das Orceïn nicht direct gebildet wird, sondern dass sich zuerst ein Körper bildet, der durch weitere Einwirkung der benützten ehemischen Agentien in den Farbstoff übergeht. Weil nun das erste Reactionsproduct in Wasser unlöslich sein konnte, so wurde absichtlich viel Salmiakgeist genommen oder Ätzkali hinzugefügt.

Die nach dem zweiten Recepte erhaltene Lösung wurde in einen geräumigen Kolben gebracht, welcher mit einem luftdicht eingesetzten Natronkalkrohr versehen wurde, um die eingedrungene Luft von der Kohlensäure zu befreien und die Bildung von Ammon- und Kalicarbonat gänzlich hintanzuhalten. Diese Fliissigkeit nahm sehr bald eine rothe Farbe an und es machte sich ebenfalls die Bildung eines braungelben Körpers und die Ausscheidung eines Farbstoffes trotz des angewendeten Ätzkalis bemerkbar. Das Ätzkali erfüllt also den angestrebten Zweck ebenfalls nicht und erschwert nur die weitere Verarbeitung, wesshalb es zweckmässiger erscheint, nur mit Ammoniak allein zu arbeiten.

Nach einem Monate bestand das Ganze aus einem dicken Farbstoffbrei und wenn man den Kolben neigte, bemerkte man, dass die Lösung nicht wie beim ersten Versuche eine blauviolette, sondern eine violettrothe Farbe besass.

Bei der weiteren Verarbeitung des durch den ersten Versuch erhaltenen Reactionsproductes wurde eine Methode befolgt, welche auf Grundlage der später gemachten Erfahrungen zweckmässiger umgestaltet wurde. Im Nachfolgenden soll also jene Methode angegeben werden, welche dermalen als die beste gilt. 
Hat man kein Ätzkali zugesetzt, so bringt man den Farbstoffbrei in einen dickwandigen, geräumigen Kolben, erwärmt denselben auf dem Wasserbade und saugt das Ammoniak mit einer Wasserstrahlpumpe grösstentheils ab. Es genügt wohl auch eine mehrstündige Erwärmung in einer auf einem Wasserbade befindlichen Schale, um die grösste Menge des Ammoniaks zu verjagen. Bei Gegenwart von Ätzkali steht zu befürchten, dass dasselbe die Farbstoffe angreifen würde, wesshalb dieser Process unterlassen werden nuss.

Der alkalische und abgektuhlte Brei wird mit Salzsäure allmählig neutralisirt, bis sich ein kleiner Überschnss dieser Säure durch einen Farbenwechsel deutlich kenntlich macht. Es entsteht hiedurch eine grosse Menge eines metallisch-schimmernden Niederschlages, der mitunter eine dentlich krystallinische Beschaffenheit zeigt. Man lässt denselben gut absitzen, filtrirt die susgefällten Farbstoffe durch ein Kattunfilter ${ }^{1}$ und wäscht den Riickstand mit Wasser so lange, bis das Filtrat keine Salzsäurereaction mehr zeigt. Dieser Rohfarbstoff enthält alle drei Farbstoffe und wird bei möglichst niederer Temperatur getrocknet.

Das Filtrat ist tief roth gefärbt und enthält den gelben Farbstoff und ziemlich viel Orceïn, welches allem Anscheine nach durch den ersteren in Lösung erhalten wird. Man engt das Filtrat auf dem Wasserbade ein und salzt die gelösten Farbsioffe durch Kochsalz aus. Der erhaltene Niederschlag wird abfiltrirt, die anhaftende Salzlauge möglichst abgesaugt und hierauf getrocknet.

Dem Rohfarbstoff, der durch Fällung mit Salzsäure und durch Aussalzen erbalten wurde, wird der beigemengte gelbe Farbstoff durch Auslaugen mit $\ddot{A}$ ther entzogen; eine Arbeit, welche etwa acht Tage dautert. Darnach wird schliesslich das vom Aussalzen herrührende Kochsalz durch Waschen mit Wasser entfernt.

Der so erhaltene Rückstand enthält Orceïn und einen in Weingeist unlöslichen Farbstoff, die man leicht von einander trennen kann. $\mathrm{Zu}$ diesem Behufe wird der Rückstand längere

1 Ich benütze seit Jahren für derlei präparative Arbeiten statt der Papierfilter Kattunfilter, welche den Druck beim Saugen aushalten und sich von dem Filtrirriickstand leicht und vollständig entfernen lassen. 
Zeit mit einer genïgenden Menge von starkem Weingeiste gekocht und der ungelöste Rückstand durch ein Faltenfilter abfiltrirt, so lange die Flitissigkeit noch heiss ist. Demselben haftet noch immer etwas Orceïn an, welches nur durch wiederholtes Auskochen hievon befreit werden kann.

Auf diese Weise gelingt es, die bei derAufeinanderwirkung des Ammoniaks und der Luft auf Orcin gebildeten Farbstoffe $\mathrm{zu}$ trennen.

Man erhält also:

a) Eine weingeistige Lösung von Orceïn,

b) eine ätherische Lösung des gelben Orcinfarbstoffes und

c) als Rückstand einen in Weingeist unlöslichen, lakmusartigen Körper.

\section{Reindarstellung des Orceïns.}

Die weingeistige Lösung des Orceïns, welche man beim Auskochen obigen Farbstoffgemenges erhielt, wird in einer Krystallisirschale auf dem Wasserbade eingeengt und in heissem Zustande mit Wasser, unter Umrïhren so weit verduinnt, bis sich an der Oberfläche goldglänzende Flecken zeigen. Es ist hiezu beiläufig. soviel Wasser nöthig, als die Lösung: misst.

Bei dieser Verdünnung fällt das Orceïn theilweise heraus und es muss ein Anwärmen vorgenommen werden, um dasselbe wieder zu lösen. Hierauf bedeckt man die Schale mit Fliesspapier und überlässt die Flüssigkeit der Krystallisation.

Nach einem Tage gesteht dieselbe zu einem dünnen Krystallbrei, der sich mit einer goldglänzenden Haut bedeckt. Man rührt denselben jeden Tag einmal durch und lässt die Krystallisation so lange währen, bis der grösste Theil des Orceïns auskrystallisirt erscheint; was man an der Farbentiefe der Mutterlauge einer kleinen Probe erkennt.

Der feinkörnige Krystallbrei wird durch ein kleines Kattunfilter, welches von dem Farbstoffe reichlich überdeckt werden muss, abfiltrirt und die anhaftende Mutterlauge desRückstandes kräftigst abgesaugt. Das feuchte Orceïn wird hierauf vom Filter entfernt und bei Zimmertemperatur im Vacuum getrocknet. Es stellt ein braunes Pulver dar, welches nur dort Metallglanz zeigt, wo es zu 
dichteren Stücken zusammengebacken erscheint. Unter dem Mikroskop stellt dasselbe ein Haufwerk von durcheinander gewachsenen Krystallen von solcher Kleinheit dar, dass man das krystallinische Gefiige nur bei bedentender Vergrösserung wahrnelmen kann. Die aus diesem Haufwerk herausragenden Spitzen lassen trotz ihrer Kleinheit wenig Licht durch, so dass es schwer hält, die Farbe dieser Substanz deutlich zu erkennen. Durch Krystallisation aus weingeistiger Lösung erhält man niemals grössere Krystalle, dagegen traten solche einmal ganz unerwartet beim Trocknen des nassen Rohfarbstoffes in einem Trockenschranke anf, welcher so stark uberheizt war, dass eine schwache Sinterung eintrat. Diese gesinterte, bronceglänzende Masse zerfiel beim Zerreiben in ein Krystallpulver, welches einem feiner krystallisirten Fuchsin glich. Unter dem Mikroskope gewahrte man ganz deutlich grössere spiessige Krystalle, welche im durchgelassenen Lichte granatroth erschienen.

Das reine krystallisirte Orceïn ist in kaltem nnd heissem Wasser unlöslich, ebenso in Äther, Benzol, Chloroform, Schwefelkohlenstoff; dagegen löslich in Aceton, Essigsäure und Weingeist. Die Lösungen zeigen eine prächtige Karminfarbe und werden durch Zusatz von Ammoniak, ätzenden und kohlensauren Alkalien prachtvoll blauviolett. Concentrirte Schwefelsäure löst in der Kälte das Orceïn zu einer blauvioletten Flïssigkeit; giesst man selbe in Wasser, so entstebt keine Fällung, sondern man erhält eine klare karminrothe Lösung, die wahrscheinlich eine Sulfosäure des Orceïns enthält. Schafwolle erhält dureh Orceïn eine schöne, amarantrothe Farbe und ist dessen Färbekraft etwa 150 bis $200 \mathrm{mal}$ so gross, wie die des Orseilleextractes.

Die Elementarzusammensetzung zweier Präparate verschiedener Abstammung war folgende:

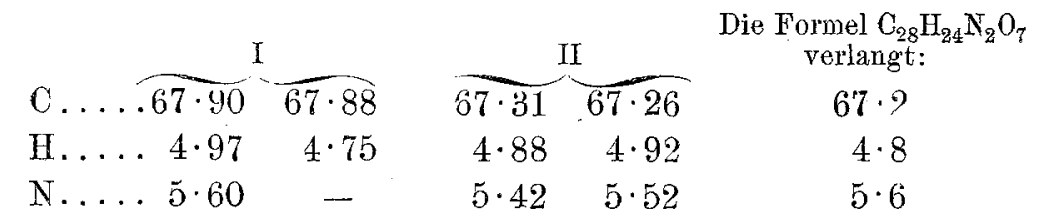

Dieser Zusammensetzung zu Folge, würde sich die Bildung des Orceïns nach folgender Gleichung gestalten:

$$
4 \mathrm{C}_{8} \mathrm{H}_{8} \mathrm{O}_{2}+2 \mathrm{NH}_{3}+6 \mathrm{O}=\mathrm{C}_{28} \mathrm{H}_{24} \mathrm{~N}_{2} \mathrm{O}_{7}+7 \mathrm{H}_{2} \mathrm{O} \text {. }
$$


Es sind bis jetzt noch keine Versuche unternommen worden, die Structurverhältnisse des Orceïns zu entziffern, doch lässt sich schon jetzt mit einiger Gewissheit vorhersagen, dass dieser Farbstoff in die Classe der Azine gehört, welche den Stickstoff in der

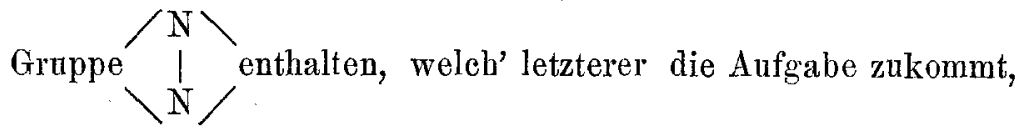
die vier Orcinreste zusammenzuhalten. Weil ferner 7 Moleküle Wasser abgespalten werden, während nur 6 Atome Sanerstoff einwirken, so ist es sehr wahrscheinlich, dass das siebente Molekül Wasser in Folge einer inneren Anhydridbildung entsteht.

Die Ausbeute an Orceïn beziffert sich auf etwa $50 \%$, bezogen auf das Gewicht des verwendeten Oreins; witrde der Bildungsprocess ganz glatt erfolgen, so müsste man $88 \%$ erhalten.

\section{Reindarstellung des gelben Orcinfarbstoffes.}

Nach der früher angegebenen Trennungsmethode erhält man denselben als ätherische Lösung, aus welcher sich schon während der Auslangung die grösste Menge an den Glaswänden abscheidet.

Zuerst wird der Äther abdestillirt und der Rückstand, dessen Menge etwa $15 \%$ des angewendeten Orcins beträgt, in kochendem Weingeist gelöst, worin er sehr leicht löslich ist. Die Lösung wird rasch durch ein Faltenfilter in eine Krystallisirsehale filtrirt und soweit mit Wasser verdünnt, bis sich an der Oberfläche das Auftreten einer metallglänzenden Haut bemerkbar macbt. Da hiedurch schon ein Theil des Earbstoffes herausgefällt erscheint, so wird die mit einem Uhrglas bedeckte Schale auf einem Wasserbade angewärmt, bis sich der Farbstoff vollkommen gelöst hat. Nun bedeckt man die Schale mit Filtrirpapier und überlässt diese Lösung der Krystallisation. Diese tritt sehr rasch ein, so dass schon nach etwa einem halben Tage das Ganze ein dichtes Haufwerk von ineinander verfilzten Krystallen darstellt und ein Umstürzen der Schale vorgenommen werden kann, ohne dass die Krystallmasse herausstürzt.

Die ausgeschiedenen Krystalle werden in einen, mit einem kleinen Kattunfilter versehenen Trichter gebracht und die tief gefärbte Mutterlauge kräftigst abgesaugt. Letztere gibt bei mehr- 
tägigem Stehen noch eine zweite Krystallisation. Die getrocknete Krystallmasse stellt ein braunes Pulver dar, welches einen griinen Metallglanz zeigt. Unter dem Mikroskope gewahrt man tief goldgelbe, mitunter gut ansgebildete Prismen.

Dieser Körper löst sich in kaltem Wasser sehr wenig, in heissem Wasser jedoch bedeutend mehr, so dass dasselbe eine ziemlich satte, orangegelbe Farbe annimmt. Von Äther wird er ebenfalls gelöst, während Orceïn bekanntlich darin unlöslich ist, was für die Trennung beider von Wichtigkeit ist. Bedeutend leichter löst sich dieser Farbstoff in Weingeist und diese Lösung besitzt eine überaus satte orangegelbe Farbe. Weun man dieselbe mit Ammoniak oder ätzenden Alkalien versetzt, so wird sie tief violett gefärbt wie eine Orceïnlösung. Mit concentrirter Schwefelsäure zusammengebracht, entsteht eine prachtvoll violette Lösung.

Durch einmaliges Krystallisiren erhält man den gelben Orcinfarbstoff nicht rein genug und es ist ein zwei- bis dreimaliges Umkrystallisiren zu seiner Reindarstellung erforderlich.

Die Elementaranalyse ergab folgender Zusammensetzung:

Die Formel $\mathrm{C}_{21} \mathrm{H}_{19} \mathrm{NO}_{5}$ verlangt:

$\begin{array}{lrr}\mathrm{C} \ldots .69 \cdot 08 & 69 \cdot 07 & 69 \cdot 04 \\ \mathrm{H} \ldots 5 \cdot 01 & 5 \cdot 02 & 5 \cdot 20 \\ \mathrm{~N} \ldots 3 \cdot 52 & 3 \cdot 80 & 3 \cdot 82\end{array}$

Dieser Zusammensetzung zufolge würde sich die Bildung dieses Farbstoffes nach folgende Gleichung gestalten:

$$
3 \mathrm{C}_{7} \mathrm{H}_{8} \mathrm{O}_{2}+\mathrm{NH}_{3}+3 \mathrm{O}=\mathrm{C}_{21} \mathrm{H}_{19} \mathrm{NO}_{5}+4 \mathrm{H}_{2} \mathrm{O} .
$$

Es sind vorlänfig keine Versuche unternommen worden, welche die Structurverhältnisse dieser Verbindung entziffern sollen, doch lässt sich schon jetzt mit einiger Wahrscheinlichkeit folgern, dass das eine Atom Stickstoff die Orcinreste zusammenhält. Weil ferner durch drei Atome Sauerstoff nicht drei, sondern vier Moleküle Wasser gebildet werden, so wird das vierte Wassermolekül wohl auch durch innere Anluydridbildung entstanden sein. 


\section{Eigenschaften des lakmusartigen Orcinfarbstoffes.}

Durch Behandlıng des mit Äther ausgelaugten Rohfarbstoffes mit kochendem Weingeiste, erhält man einen unlöslichen Rückstand, dessen Menge auf etwa $15 \%$ veranschlagt werden kann, und welchem man durch wiederholtes Auskochen das etwa beigemengte Orceïn entziehen kann.

Weil dieser Körper amorph, in allen gewöhnlichen Lösungsmitteln unlöslich und dessen Ausbeute gering ist, so hat die bisherige Untersuchung keine abschliessenden Ergebnisse hinsichtlich seiner Zusammensetzung ergeben.

Diese Substanz zeigt einen grïnen Metallganz, löst sich in Ammoniak, in ätzenden und kohlensauren Alkalien mit dunkelblauer Farbe auf. Tropft man diese Lösung in Wasser ein, so nimmt dasselbe genan diejenige Farbe an, welche eine Lakmuslösung demselben ertheilt und setzt man hierauf eine Säure hinzu, so wird die Flüssigkeit zwiebelroth; gerade so, als ob man obigen Jndicator verwendet hätte.

In der angesäuerten, zwiebelrothen Flüssigkeit ist der Farbstoff nicht gelöst, denn nach längerem Stehen setzt sich derselbe in feinen kirschrothen Flöckchen ab.

Es lag der Gedanke nahe, dass dieser Farbstoff mit dem Lakmusfarbstoff identisch ist, weil derselbe ebenfalls in den gewöhnlichen Lösungsmitteln nnlöslich ist. Um diese Frage zn beantworten, hat der zweitgenannte Verfasser dieses Aufsatzes die Farbstoffe des käuflichen Lakmus in Untersuchung gezogen, welche indess noch nicht abgeschlossen ist, so dass sich hierüber noch nichts Sicheres sagen lässt.

Wenn man einer ammoniakalischen und filtrirten Lösung dieser Substanz den Ammoniaküberschnss durch Erwärmen oder durch Stehenlassen im Vacuum über Schwefelsäure, entzieht, so nimmt dieselbe nach und nach eine immer röthere und schliesslich eine kirschrothe Farbe an; ein Beweis, dass eine saure und neutrale Ammonverbindung existiren muss. Diese Elüssigkeit wird dicklich wie eine Gallerte und trocknet schliesslich zu einer metallglänzenden, in Wasser löslichen Masse ein.

Wenn man eine alkalische und filtrirte Lösung dieses Orcinfarbstoffes mit einer Säure neutralisirt, so fällt derselbe in 
ausserordentlich feinen, braunrothen Flöckchen heraus, welche sich erst nach tagelangem Stehen absetzen. Die Filtration dieses Niederschlages macht die grösste Mülue und erfordert sehr viel Zeit. Der abfiltrirte und gewaschene Niederschlag trocknet zu einer ziemlich harten, formlosen, metallisch grünen Masse ein, welche zwar analysirt wurde, aber gar keine übereinstimmenden Ergebnisse geliefert hat.

Auf die bisherige Art ist dieser Substanz nicht beizukommen und es mïssten verschiedene Derivate derselben hergestellt werden, von denen wahrscheinlich eine oder die andere Verbindung Aufschluss îber die Zusammensetzung und Entstehung geben dürfte. Fủl solche Versuche ist uns das Material ausgegangen und es bleibt die Ergänzung dieser Arbeit einem späteren, günstigeren Zeitpunkte vorbehalten.

\section{Über die Einwirkung der Luft anf eine alkalische Lösung. des 0rcins.}

Um zu entscheiden, ob das Orcein picht vielleicht aus einem Oxydationsproducte des Orcins gebildet werde, wurde eine wässerige Orcinlösung mit Ätzkali versetzt und einige Wochen der Luft dargeboten. Die tiefbraun gewordene Flissigkeit wurde mit einer Säure neutralisirt, ohne dass hiedurch ein Niederschlag entstand. Der Abdampfrückstand dieser angesäuerten Lösung löste sich in Wasser und Weingeist mit braungelber Farbe auf. Diese Lösungen zeigten eine schwache Fluorescenz, welche auf Zusatz von Ätzkali oder Ammoniak stärker hervortrat. Durch Einwirkung von Ammoniak konnte kein Orceïn oder sonst irgend welcher Earbstoff erhalten werden. Es ist sehr leicht möglich, dass der im Lakmus vorkommende fluorescirende Körper mit obiger Verbindung identisch ist.

\section{Über die Einwirkung von Ammoniak und Wasserstoff- hyperoxyd anf orcin.}

Es wurde schon Eingangs elwähnt, dass der Luftsauerstoff durch Wasserstoffhyperoxyd ersetzt werden kann und dass in diesem Falle der Process weit rascher verläuft. Audere Oxydationsmittel wie z. B. Chlorkalk, Kaliumpermanganat wurden zwar auch versucht, aber mit sehr ungünstigem Erfolge. 
Nachdem die Molekularformel des Orceïns einmal festgestellt war, so liess sich sehr leicht das Verhältniss der aufeinanderwirkenden Stoffe berechnen. Aus der Bildungsgleichung $\underset{\text { kryst. Orcin }}{4\left(\mathrm{C}_{7} \mathrm{H}_{8} \mathrm{O}_{2}+2 \mathrm{aq}\right)}+2 \mathrm{NH}_{3}+6 \mathrm{H}_{2} \mathrm{O}_{2}=\underset{28}{\mathrm{C}_{28}} \underset{\mathrm{H}_{24} \mathrm{~N}_{2} \mathrm{O}_{7}}{\text { Orceïn }}-21 \mathrm{H}_{2} \mathrm{O}$ folgt, dass man zu nehmen habe:

Krystallisirtes Orein........100 Gewichtstheile

Ammoniak zu $100 \% \ldots \ldots .6 .6 \%$ oder:

Wasserstoffsuperoxyd zu $100 \% \cdot 35 \quad "$

Krystallisirtes Orcin ......... 100 "

Salmiakgeist zu $22 \% \ldots \ldots \ldots 27 \cdot 3$ "

Wasserstoff hyperoxyd zu $3 \% \ldots 1197$ "

Weil aber das Ammoniak auch als Lösungsmittel zu wirken hat, so muss ein beträchtlicher Überschuss genommen werden, wenn man nicht vorzieht, statt dessen Ätzkali zu rerwenden, welches aber keinen Vortheil darbietet. Es wurde daher nach folgendem, mehrfach erprobten Recepte gearbeitet:

Krystallisirtes Orcin ........100 Gewichtstheile

Salmiakgeist zu $22 \% \ldots \ldots \ldots 200$ n

Wasserstoffsuperoxyd zu $3 \% \ldots 1200 \quad$ "

Man löst das gepulverte Orcin in einem Kolben in dem Salmiakgeiste auf und giesst das Wasserstoffsuperoxyd hinzu. Es tritt sofort eine braunrothe Färbung ein, welche an Farbentiefe lang:sam zunimmt; es macht sich ferner eine Erwärmung bemerkbar, die schliesslich eine Temperatur von $60^{\circ}$ erreicht. So wie die Erhitzung eintritt, findet ein allmählicher Farbenwechsel statt; das Braunroth geht in ein sattes Karminroth, und dieses in ein Violett über. Den nächsten Tag stellt das Ganze einen violetten, körnigen Farbbrei dar, welcher mit goldglänzeuden Häutchen bedeckt ist.

Da nach der Erzeugungsgleihcung der Wasserstoff des Ammoniaks oxydirt werden muss, so ist ein Überschuss desselben bedenklich, weil das Wasserstoffhyperoxyd möglicherweise zu einer Nebenreaction verbraucht werden könnte. Es wurde daher der Versuch in der Weise abgeändert, dass man nur die theoretisehe Menge von Ammoniak verwendete und als Lösung:s- 
mittel für den gebildeten Farbstoff, Ätrkali hinzufügte. Diese Abänderung brachte nur den einzigen bemerkenswerthen Unterschied hervor, dass der Farbbrei eine röthere Farbe zeigte; ein Vortheil liess sich nicht erkennen.

Allem Anscheine nach ist die Reaction in einem Tage nicht ganz beendet, denn der Farbbrei nimmt an Farbentiefe und Dickfliissigkeit zu, so dass das Gemisch erst wach drei bis vier Tagen den Reifezustand erreicht zu haben scheint.

Die Verarbeitung des alkalischen Farbbreies erfolgte genau so, als ob derselbe durch Luftoxydation erhalten worden wäre, denn auch in diesem Falle werden durch mehrere Nebenreactionen die früher beschriebenen Farbstoffe als Nebenproducte gebildet; auch ist die Ausbeute so ziemlich dieselbe.

Die Elementaranalyse des mittelst Wasserstoffhyperoxyd erhaltenen Oreë̈ns lieferte bei drei Präparaten verschiedener Abstammung folgende Zablen:

$\begin{array}{cccc}a & b & \stackrel{e}{D} & \begin{array}{c}\text { Die Formel } \mathrm{C}_{28} \mathrm{H}_{24} \mathrm{~N}_{2} \mathrm{O}_{7} \\ \text { verlangt: }\end{array} \\ \mathrm{C} \ldots \ldots 66 \cdot 43 & 66 \cdot 41 & 67 \cdot 03 & 67 \cdot 2 \\ \mathrm{H} \ldots \ldots 4 \cdot 69 & 4 \cdot 81 & 4 \cdot 85 & 4 \cdot 8 \\ \mathrm{~N} \ldots \ldots 5 \cdot 67 & 5 \cdot 47 & 5 \cdot 38 & 5 \cdot 6\end{array}$

Die übrigen zwei Farbstoffe wurden keiner Untersuchung unterzogen, weil sie in allen ihren Eigenschaften mit den durch Luftoxydation erhaltenen übereinstimmten und an deren Identität nicht gezweifelt werden konnte.

\section{Einwirkung von Ammoniak und Wasserstoffhyperoxyd auf einige andere mehrwerthige Phenole.}

Resorcin. Es ist bereits bekannt, dass eine mit Ammoniak und Soda versetzte Resorcinlösung an der Luft in einigen Tagen eine blaue Farbe annimmt, und dass aus dieser Flïssigkeit mit Säuren ein angeblich orceïnartiger, metallisch glänzender Körper ausgefällt werden soll.

Wir haben diese Reaction geprüft in der Weise, dass wir das Resorcin derselben Behandlung mit Ammoniak und Wasserstoffhyperoxyd unterzogen haben, wie das Orcin. Bei diesem Processe findet ebenfalls eine Erwärmung statt, die Flüssigkeit nimmt aber eine graublaue Farbe an und es setzt sich am Boden 
ein dunkler, feiner Niederschlag ab. Lässt man das Ganze einige Tage stehen, bis sich keine weitere Veränderung bemerkbar macht, so fällt eine Säure aus dieser Flüssigkeit eine dunkle Substanz heraus, welche im gewaschenen und getrockneten Zustande eine braune Farbe mit kaum wahrnehmbarem Metallglanz zeigt.

In Weingeist ist dieselbe schwer löslich mit schmutzigrother Farbe; in Wasser ganz unlöslich, ebenso in Äther und Chloroform. Dagegen löst sich der Körper in concentrirtem Ammoniak und ätzenden Alkalien mit indigoblauer Farbe, welche indess nach einiger Zeit missfarbig wird. Derselbe ist nach diesen Ergebnissen mit dem Orceïn gar nicht verwandt, sondern ähnelt eher dem lakmusähnlichen Farbstoff, der aus Orcin als Nebenproduct erhalten wird.

Eine nähere Untersuchung dieser Substanz wurde nicht vorgenommen.

Resorcin und Orcin. Ganz anders gestalten sich die Verhältnisse, wenn man das Resorcin mit Orcin combinirt; denn in diesem Falle wird wirklich ein orceïnartiger Körper, ein gemischtes Orceïn gebildet, welches als Reso-Orceïn benannt werden mag. Nimmt man an, dass der Bildungsprocess in derselben Weise verläuft wie bei Oreeïn, so hat man anf zwei Moleküle Orcin, 2 Moleküle Resorcin, 2 Moleküle Ammoniak und 6 Moleküle Wasserstoffhyperoxyd zu nehmen, woraus sich folgendes Recept ergibt:

Gewichtstheile

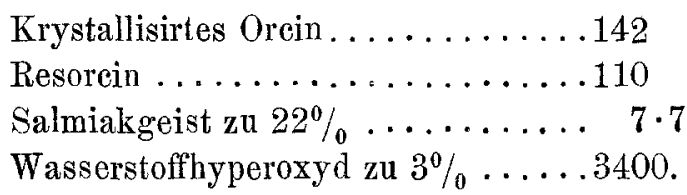

Da das Ammoniak auch die Bestimmung hat, als Lösungs. mittel zu wirken, so hat man beiläufig doppelt soviel Salmiakgeist zu nehmen, als das Gewicht des Orcins und Resorcins beträgt.

Wenn man die beiden Phenole in dem Salmiakgeiste löst, hernach das Wasserstoffhyperoxyd hinzufügt, so tritt sofort eine Bräunung der Flüssigkeit ein, welche Farbe nach und nach röther wird. Die Temperatur steigt und so wie diese Erwärmung 
eintritt, wird die Flüssigkeit tief blauviolett und schliesslich reinblau. Das auf diese Art erhaltene Reactionsproduct wurde nach mehrtägigem Stehen durch Erwärmung und Absaugen von dem grösseren Theil des Ammoniaks befreit und der gebildete Farbstoff mit Salzsäure gefällt. Der erhaltene Niederschlag wurde abfitrirt und gewaschen, wobei das Filtrat eine sehmutzigrothe, aber nicht sehr tiefe Farbe zeigte. Der Abdampfrickstand dieses Filtrats war gering und bestand ats einer schmierigen Masse, die sich in Weingeist mit schmutzigrother Farbe löste und mit Ammoniak eine sehmutzigviolette Farbe annahm. Die Menge des gereinigten und getrockneten Rohfarbstoffes bezifferte sich auf $73 \cdot 4 \%$, während die Theorie eine Ausbeute von $93 \cdot 6 \%$ verlangt.

Dieser Rohfarbstoff wurde in Weingeist ansgekocht und die erhaltene, tief fuchsinrothe Lösung in eine Krystallisirschale filtrirt, mit Wasser bis zur beginnenden Ausscheidung verdünnt, nachher angewärmt und der Krystallisation überlassen. Bei dieser Behandlung blieb ein in Weingeist unlöslicher Rückstand übrig, dessen Menge etwa 18\% betrug und welcher in seinen Eigenschaften ebenfalls dem Lakmus gleicht.

Aus der weingeistigen Lösung krystallisirte der Farbstoff langsam heraus, während sich die Flüssigkeitsoberfläche mit einer bronceglänzenden Sehichte bedeckte.

Der auskrystallisirte Farbstoff wurde von der Mutterlauge durch Filtration und Absaugen befreit und schliesslich getrocknet. Er stellt bronceglänzende Brocken dar, welche unter dem Mikroskope ein dentlich krystallinisches Gefüge verrathen und welche sich schon in schwachem Weingeist sehr leicht lösen, während das Orceïn darin entschieden schwerer löslich ist. Die weingeistige Lösung hat eine ungemein tiefe, prachtrolle Fuchsinfarbe, ist also entschieden blauer als eine Orceïnlösung und nimmt durch Zusatz von ätzenden Alkalien oder Ammoniak eine reinblaue Farbe an. In concentrirter Schwefelsäure löst sich das ResoOrceïn mit prachtroll blauer Farbe auf, während das Orceïn eine violette Lösung liefert.

Wenn der Bildungsprocess dieses Farbstoffes in gleicher Weise stattfindet wie bei Orceïn, so müsste derselbe nach folgender Gleichung erfolgen: 


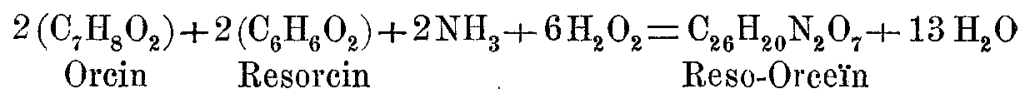

und die Zusammensetzung des gebildeten Farbstoffes der in dieser Gleichung enthaltenen Molecularformel entsprechen. Die Elementaranalyse bestätige diese Voraussetzung, denn sie ergab folgende Zahlen:

$\begin{array}{ccc} & & \begin{array}{c}\text { Die Formel } \mathrm{C}_{26} \mathrm{H}_{20} \mathrm{~N}_{2} \mathrm{O}_{7} \\ \text { verlangt: }\end{array} \\ \mathrm{C} \ldots .65 \cdot 57 & 65 \cdot 82 & 66 \cdot 08 \\ \mathrm{H} \ldots 4 \cdot 39 & 4 \cdot 34 & 4 \cdot 24 \\ \mathrm{~N} \ldots 5 \cdot 79 & 5 \cdot 65 & 5 \cdot 95\end{array}$

Orcin und andere mehrwerthige Phenole. Es wurden ron uns fast alle Dioxy- und Trioxyderivate des Benzols hinsichtlich ihres Verhaltens gegen Ammoniak und Wasserstoffhyperoxyd untersucht und es gab kein einziges dieser Phenole für sich allein, irgend einen befriedigenden Erfolg. Es entstanden zumeist braune Reactionsproducte, die gar keine, zur weiteren Untersuchung verlockenden Eigenschaften besassen. Auch wenn man diese Phenole mit Orcin combinirt, so kommt nicht viel dabei heraus, obwohl die entstehenden Verbindungen noch eher als „Farbstoffe" gelten könnten.

Schlusswort. Nachdem das Orceïn seine einstige Bedeutung in der Färberei, durch die zahlreichen Producte der Theerfarbenindustrie stark eingebüsst hat, so sind die Ergebnisse vorliegender Arbeit kaum geeignet, die Fabrication der bisherigen Orseillefarbstoffe zu beleben oder zu einer Vereinfachung der künstlichen Darstellung des Orcins aufzumuntern. Sie hat aber doch die eine nicht zu unterschätzende Aufgabe gelöst: Die wahre Zusammenzetzung dieses Farbstoffes und den Bildungsprocess desselben festgestellt zu haben; auch lässt sich schon jetzt eine gewisse Beziehung desselben zu manchen anderen künstlichen Farbstoffen deutlich erkennen.

Diese Arbeit bedarf noch der Ergänzung durch die Herstellung verschiedener Orceïnderivate, welche die Structur des Orceïns deutlich erkennen lassen und ebenso auch eine Ergänzung in Bezug anf die Nebenproducte seiner Darstellung. Nach- 
dem durch allerlei Berufsgeschäfte eine Pause in der Fortsetzung dieser Arbeit eintreten musste, so soll das Fehlende später nachgetragen werden.

Die bisherigen Erfolge dieser Untersuchung baben auch wichtige Fingerzeige über die Entstehung des gelben Orcinfarbstoffes geliefert und es ist bereits möglich geworden aus Orcin auf eine sehr einfache Art einen Farbstoff herzustellen, welcher die Hauptrenctionen des ersteren zeigt. Die Untersuchung hierïber dürfte allem Anscheine nach zu wichtigen Resultaten führen. 